mapping (modified look-locker) to estimate extracellular volume (ECV), T2 mapping (to quantify oedema) and strain analysis. A panel of circulating biomarkers (including inflammatory, ischaemic and fibrosis markers) was evaluated by multiplex ELISA. Longitudinal histological analysis was performed in the rats and correlated with imaging and biomarker findings.

Results Left ventricular function (LVEF) declined steadily during treatment in rats and humans. Persistent LV dysfunction was seen in $23 \%$ of patients 12 months after therapy. Peak Troponin I levels correlated with fall in (LVEF) and circulating levels translated well between rat and humans. However, levels did not peak until the final cycle of chemotherapy when significant LV decline had already occurred. There was no significant change in the other circulating biomarkers. ECV did not change significantly during treatment but lower baseline ECV correlated with a greater fall in LVEF. Microscopic changes were seen in the rat myocardium after 6 doses of doxorubicin but electron-microscopy revealed mitochondrial damage after just one dose.

Conclusions This translational approach enabled forward and back translation leading to the development of a clinically relevant pre-clinical cardiotoxicity model. Troponin I was the most informative circulating biomarker but peaked at the end of therapy too late to modify treatment and prevent LV decline. The model has the potential to be used to identify earlier biomarkers and evaluate cardioprotective strategies. The imaging findings showed that fibrosis cannot be detected on CMR within one year of chemotherapy but generated a new hypothesis that patients with 'healthier hearts' may be a greatest risk of drug-induced cardiotoxicity.

\section{COMPREHENSIVE CARDIOVASCULAR MAGNETIC RESONANCE ASSESSMENT OF ANDERSON-FABRY CARDIOMYOPATHY- NATURAL HISTORY AND ASSESSMENT OF TREATMENT EFFECT}

Anna Reid, Chris Miller, Ana Jovanovic, Peter Woolfson, Nik Abidin, David Clark, Wendy Gamlin, Glyn Coutts, Matthias Schmitt.

\subsection{6/heartjnl-2017-311399.20}

Background The Anderson Fabry Cardiomyopathy (AFC) is heterogeneous, complex, and its pathophysiology remains incompletely understood. There is uncertainty regarding the optimal timing and impact of enzyme replacement therapy (ERT) in cardiac involvement, with a pressing need for biomarkers able to identify early disease, monitor and guide treatment.

Objectives The aims of this study were two-fold: 1) to perform comprehensive cardiac phenotyping in a large cohort of Anderson-Fabry disease (AFD), and 2) characterise their shortterm natural history and assess the impact of ERT using multiparametric Cardiac Magnetic resonance imaging (MPCMRi), echocardiography and ECG analysis.

Methods 113 prospectively enrolled, genetically-confirmed, AFD patients were compared to 20 age-matched healthy volunteers (HVs). 37 of these patients re-attended for a one-year follow-up scan, and were sub-grouped according to treatment status (newly started on ERT, ERT naïve, and established on ERT).

Results The AFC is characterised by increased LV mass of several different hypertrophic patterns, impaired LV global longitudinal strain and left atrial function, low myocardial native T1, high T2, and increased extracellular volume fraction. Significantly higher T1s were seen in the inferolateral wall, even before the development of increased wall thickness or LV mass, or LGE. A newly proposed marker of disease severity, the ' $T 1$ ratio', reflects the relationship between inferolateral and remote myocardial $\mathrm{T} 1$, and strongly correlated with LV mass, percentage LGE and ECV fraction, whilst avoiding potential uncertainty caused by pseudo-normalisation of $\mathrm{T} 1 \mathrm{~s}$ in severe AFD.

ERT initiation reduced LVM. T1 values (excluding the inferolateral wall) fell significantly in untreated patients (974 $\pm 36 \mathrm{~ms}$ v $955 \pm 44 \mathrm{~ms}, \mathrm{p}=0.04)$ associated with a significant increase in voxel based spread ( $288 \mathrm{v} 350, \mathrm{p}=0.02)$, whereas both treatment groups showed no change in $\mathrm{T} 1$ values. The T1 ratio showed a trend towards improvement $(0.94 \pm 0.08 \mathrm{v}$ $0.98 \pm 0.1, \mathrm{p}=0.05)$ in the established ERT group. Electrocardiography and echocardiography did not detect disease progression or treatment effects in any group.

Conclusions AFC is a complex pathology with intracellular and extracelluar disease components. MPCMRi elucidates these disease processes and allows early disease detection, and possibly disease and treatment monitoring.

\section{PERFUSION CARDIOVASCULAR MAGNETIC RESONANCE (CMR) - CAN DAVID (RESOLUTION) TAKE ON GOLIATH (COVERAGE) AGAIN?}

${ }^{1}$ Joy S Shome, ${ }^{2}$ Kerem C Tezcan, 'Sohaib Nazir, ${ }^{1}$ Markus Henningsson, ${ }^{1}$ Adriana Villa, ${ }^{1}$ Imran Rashid, 'David Snell, ${ }^{4}$ Antonis Pavlidis, ${ }^{4}$ Brian Clapp, ${ }^{3,4}$ Simon Redwood, ${ }^{5}$ Kamran Baig, ${ }^{1}$ Reza Razavi, ${ }^{1,4}$ Tevfik F Ismail, ${ }^{1}$ Amedeo Chiribiri, ${ }^{3,4}$ Divaka Perera, ${ }^{2}$ Sebastian Kozerke, 1,6Sven Plein. 'Division of Imaging Sciences, The Rayne Institute, King's College London, St. Thomas' Hospital, London, UK; ${ }^{2}$ Department of Biomedical Engineering, ETH Zurich, Zurich, Switzerland; ${ }^{3}$ Cardiovascular Division, The Rayne Institute, King's College London, St. Thomas' Hospital, London, UK; ${ }^{4}$ Department of Cardiology, St. Thomas' Hospital, London, UK; ${ }^{5}$ Department of Cardiothoracic Surgery, St. Thomas' Hospital, London, UK; ${ }^{6}$ Leeds Institute of Cardiovascular and Metabolic Medicine, University of Leeds, Leeds, UK

\subsection{6/heartjnl-2017-311399.21}

Background Both 3D and high-resolution 2D-perfusion CMR accurately detect coronary artery disease (CAD). 3D provides whole-heart coverage whereas $2 \mathrm{D}$ better detects sub-endocardial ischaemia. We compared the diagnostic accuracy of both techniques to detect flow limiting $\mathrm{CAD}$ as measured by fractional flow reserve (FFR). We also investigated the relative accuracy of these tools in identifying prognostically significant myocardial ischaemic burden (MIB).

Methods Patients with suspected angina underwent high spatial resolution 2D $k$ - $t$ SENSE (3 slices, in-plane spatial resolution $1.3 \times 1.3 \times 8 \mathrm{~mm})$ and $3 \mathrm{D} k-t$ PCA whole heart $(12$ slices, in plane spatial resolution $2.3 \times 2.3 \times 5 \mathrm{~mm})$ myocardial perfusion CMR during adenosine stress in a single sitting. Invasive coronary angiography with FFR (for stenoses of 50-80\% severity visually) was performed in all patients prior to revascularisation. Perfusion defects were contoured using circleCVI software and MIB was calculated for both $2 \mathrm{D}$ and $3 \mathrm{D}-\mathrm{CMR}$. The anatomical and functional BCIS-1 Jeopardy Scores (BCIS-JS) scores were calculated from the invasive angiograms. Results Forty-seven patients were included in the analysis. Per-patient sensitivity, specificity, diagnostic accuracy, PPV, and NPV in identifying flow-limiting CAD were 76\%, 100\%, $85 \%, 100 \%, 72 \%$ for $2 \mathrm{D}$ 